\title{
Principes fondamentaux et concept de travail de la médecine de la personne âgée Profil de la gériatrie en Suisse*
}

\author{
Avant-propos \\ Daniel Grob, président de la SPSG 2005/2006
}

En Suisse, la gériatrie est en plein développement et c'est en raison du vieillissement de la population que de nombreuses institutions gériatriques sont progressivement mises en place. Toutefois, ces institutions, apparues dans les cantons et les communes au cours de plusieurs dizaines d'années, peuvent être très différentes les unes des autres.

La gériatrie est une spécialité médicale. L'Organisation mondiale de la santé la définit ainsi: «La gériatrie est la branche de la médecine qui s'occupe de la santé à un âge avancé ainsi que des aspects cliniques, de prévention, de réadaptation et des aspects sociaux des maladies des personnes âgées.»

Gériatrie signifie également travail interdisciplinaire car plusieurs professionnels de la santé doivent travailler ensemble en étroite collaboration dans une optique gériatrique. Un tel travail d'équipe est souhaitable et nécessaire et ne peut fonctionner que si chaque corps de métier possède une identité propre tout en respectant également celle de l'autre.

C'est pour cela que la Société Professionnelle Suisse de Gériatrie (SPSG) - c'est-à-dire l'organisation professionnelle des médecins gériatres en Suisse - présente dans ce document le profil de la gériatrie. Il devrait aider tous ceux qui sont impliqués à la construction des structures gériatriques. Il reflète de manière réaliste la vision de médecins gériatres qui s'occupent de la prise en charge des personnes âgées souvent depuis des décennies.

La première partie de ce document contient les bases fondamentales et les concepts de travail de la médecine de la personne âgée. La seconde partie - encore en cours de réalisation - présentera les critères de la prise en charge gériatrique à l'intention des structures, pour l'établissement des procédures et pour l'obtention des résultats (outcome).

La réalisation de ce document est le fruit de plusieurs années de travail et d'intenses discussions au sein du groupe de travail ad hoc ainsi que parmi le Comité de la SPSG.

Des remerciements particuliers vont aux membres du groupe de travail initial: PD Dr Christophe Büla, Epalinges; Dr Ruedi Gilgen, Zurich; Dr Kurt Müller, Schaffhouse et PD Dr Philippe Huber, Genève. De plus, les Dr Charles Chappuis, Berne; Dr Franco Tanzi, Lugano; Dr Regula Schmit-Mannhart, Berne; PD Dr Albert Wettstein, Zurich et PD Dr Gilbert Zulian, Genève ont participé de manière significative à la rédaction de ce document.

Des remerciements vont aussi au Pr Andreas Stuck, Berne pour ses suggestions pertinentes. Finalement, un tout grand merci à Mme Pia Graf Voegeli, responsable du secrétariat de la SPSG, qui a coordonné l'ensemble des travaux de rédaction.

La traduction et l'adaptation française de ce document ont été effectuées par Mme le Dr Rebecca Dreher-Bruggmann (Aubonne et Epalinges) avec l'aide de PD Dr Gilbert Zulian.

Ce document a été approuvé dans sa version présente lors de l'Assemblée générale de la SPSG tenue le 9 novembre 2006 à Schaffhouse.

Le comité de la SPSG accepte volontiers toutes les suggestions qui lui seront faites et reste entièrement à disposition.

\section{Introduction}

La Société Professionnelle Suisse de Gériatrie est l'organisation faîtière des médecins gériatres en Suisse. Elle a été fondée en l'an 2000 après que la Fédération des médecins suisses (FMH) eut établi la formation approfondie de médecin gériatre.

Dans le cadre de la $\mathrm{FMH}$, la gériatrie est en effet reconnue en tant que formation approfon-
Correspondance: Secrétariat SFGG/SPSG Spital Bern-Ziegler CH-3001 Berne Tél. 0319707798 Fax 0319707805
La SPSG réunit, en tant qu'association professionnelle permanente, les médecins internistes FMH ou généralistes FMH avec spécialisation en gériatrie (= membres ordinaires), les médecins pendant la formation pour la spécialisation (= membres temporaires) et les autres praticiens intéressés à la gériatrie (= membres associés). Elle a été fondée en 1992 sous le toit de la SGG-SSG et depuis le $1^{\mathrm{er}}$ janvier 2003 elle est une société indépendante avec des propres statuts. Pour informations ultérieures: www.geriatrie-suisse.ch. 
die: les médecins gériatres sont des médecins généralistes ou des médecins internistes qui ont accompli au total pas moins de 7 ans de formation postgraduée, dont 3 en gériatrie et psychogériatrie.

Par rapport à d'autres pays européens, la reconnaissance de la formation continue approfondie en gériatrie s'est produite relativement tardivement. C'est le vieillissement démographique qui a été le moteur de son développement étant données les perspectives d'accroissement du nombre de personnes âgées et le besoin d'une prise en charge médicale spécialisée avec des soins et des traitements spécifiques. ${ }^{\mathrm{a}}$

On s'attend à ce que le nombre de personnes âgées de plus de 80 ans augmente de $65 \%$ d'ici à 2030 en Suisse. Mais en fait, seule une minorité de ces personnes est dépendante et nécessite par conséquent de l'aide. Environ 10\% des personnes âgées de 80 ans vivent en institutions de long séjour et en EMS, près de $20 \%$ à l'âge de 85 ans et même $40 \%$ à l'âge de 90 ans. Il en résulte une augmentation des coûts par le besoin en personnel lié à l'institutionnalisation ce qui entraîne à son tour une augmentation globale des coûts.

Cette pression prévisible sur les coûts est un défi pour les personnes travaillant en gériatrie. Mais le développement vertigineux de la médecine présente un autre défi car les grands vieillards pourront également profiter des progrès dans les investigations et les traitements médicaux. Le développement de la santé publique, tout particulièrement en milieu hospitalier pour y réduire la durée de séjour, met sous pression toutes les institutions gériatriques rattachées à un hôpital de soins aigus. Dans ce contexte, il est particulièrement important de développer des structures gériatriques qui maintiennent des soins de qualité dans la prise en charge du grand vieillard alors que le contexte du système de santé est en fait plus axé sur les besoins des personnes plus jeunes et plus indépendantes.

La gériatrie consiste en une médecine globale de la personne âgée et tout particulièrement du grand vieillard. De ce fait, son devoir principal est de promouvoir une prise en charge adaptée afin que la personne âgée puisse vivre de manière indépendante le plus longtemps possible. Ceci ne résulte pas seulement de raisons économiques mais bien plus de la volonté exprimée par la grande majorité de la population âgée («primauté à l'autonomie»). Une expertise médicale gériatrique doit donc être exigée dans les milieux ambulatoires, à l'hôpital et en institutions de long séjour afin d'offrir la plus haute qualité possible de traitements et de prises en charge.
Dans ce sens, la gériatrie n'est pas que de la médecine dans le sens curatif du terme car la spécialité gériatrique comprend des mesures de prévention et de réadaptation, des mesures sociales et des mesures palliatives. Elle consiste en une médecine globale pour des personnes âgées qui souffrent de maladies chroniques et qui sont souvent polymorbides et dépendantes.

\section{Définitions et concepts de la gériatrie}

\subsection{Définitions}

La gériatrie est la branche de la médecine qui s'occupe de la santé à un âge avancé, ainsi que de tous les aspects cliniques (physiques et psychiques) de prévention, de réadaptation et des aspects sociaux des maladies des personnes âgées.

Cela signifie donc une prise en charge comportant des traitements curatifs et préventifs, des traitements de réadaptation et de palliation qui incluent les aspects sociaux.

Ces entités sont d'ailleurs souvent présentes de manière concomitante et la distinction entre les unes et les autres n'est pas toujours claire.

\subsection{Le médecin gériatre}

Le médecin gériatre est spécialisé en médecine générale ou en médecine interne. Il a de plus accompli une formation approfondie d'au minimum 3 ans en gériatrie et en (géronto-)psychiatrie et il a réussi l'examen professionnel de gériatrie.

Le médecin gériatre possède un savoir, un savoir-faire et un savoir-être dans les domaines suivants [2]:

- prendre en compte le fait que les maladies de la personne âgée sont souvent multiples, complexes et chroniques et qu'elles s'influencent mutuellement;

- connaître les facteurs qui sont dépendants de l'âge et leur influence sur la santé, la maladie, le corps et le psychisme; connaître les symptômes et les réactions différentes ainsi que les réserves diminuées de l'organisme; ${ }^{\text {b }}$

- connaître les symptômes et les conséquences de la polymorbidité (existence de maladies concomitantes, respectivement invalidantes);

- connaître les facteurs de risque à l'âge avancé, les principes et les possibilités de prévention;

- apporter des soins aux personnes âgées ayant des fonctions cérébrales réduites entraînant des problèmes médico-sociaux;

- connaître les traitements et les prises en charge de réadaptation des maladies aiguës et chroniques de la personne âgée;

- utiliser les concepts de la médecine palliative chez la personne âgée;

- accompagner les personnes en fin de vie; an Suisse voir [1]

b Concept de fragilité (frailty): [3]. 
- posséder des capacités de communication avec les personnes âgées, également dans des conditions difficiles;

- intégrer et soutenir l'entourage lors de la prise en charge;

- savoir travailler avec les organes de la santé publique;

- savoir formuler des objectifs gériatriques interdisciplinaires et être responsables de leur explication;

- transmettre le savoir, le savoir-faire et le savoirêtre gériatrique;

- élaborer des projets scientifiques ou cliniques y compris en interdisciplinarité.

\subsection{Objectif de la prise en charge gériatrique} La prise en charge gériatrique a comme objectif de permettre à la personne âgée une vie pleine et active, de prévenir les maladies et leur suite ou de les diagnostiquer et de les traiter précocement. Elle a comme objectif supplémentaire d'offrir une vie réussie aux personnes âgées qui souffrent de maladies chroniques ou aiguës, qu'elles soient dépendantes ou atteintes dans leur fonctionnalité. Elle fournit aux malades en fin de vie le soutien médical et le soutien social nécessaire ainsi qu'un accompagnement.

Le lien obligé de l'évaluation gériatrique compréhensive, en tant que méthode diagnostique, avec la planification de la prise en charge ont été maintes fois démontrés dans la littérature scientifique comme une condition nécessaire à l'efficacité de la prise en charge globale. Pour cela, nous nous référons aux références suivantes: [7-11].

d L'évaluation gériatrique compréhensive repose sur le modèle et sur le concept de la classification internationale de la capacité fonctionnelle, de l'invalidité et de la santé (ICF): l'évaluation médicale classique de la maladie qui repose sur une pathologie ou sur un dysfonctionnement est élargie en tenant compte des handicaps et des répercussions sur le bon fonctionnement social. Les maladies chroniques et irréversibles fréquentes chez les personnes âgées - peuvent entraîner comme conséquence une menace de perte d'autonomie dans les activités de la vie quotidienne de base et dans les activités instrumentale ce qui influencera la qualité de la vie. Dans le modèle ICF sont incorporées les dimensions des fonctions corporelles (bonne santé et maladie), celles des activités d'une personne (activités de la vie quotidienne) ainsi que l'implication dans un contexte social (vie associative) et des facteurs contextuels.

\subsection{Principes éthiques}

L'exigence du respect de la dignité est inconditionnelle pour toute personne humaine.

Ce principe de base signifie le droit à la protection et le droit à l'autonomie de la personne. Or, la diminution de l'autonomie est plus fréquente à l'âge avancé et cela peut entraîner un déséquilibre entre la dépendance et l'indépendance d'une personne. La personne âgée est alors particulièrement vulnérable mais cela ne diminue pas pour autant l'exigence de respecter sa dignité, sa protection et son autonomie. C'est pourquoi il faut apporter la plus grande attention à la protection et à la prise en charge des personnes âgées.

Les cinq aspects suivants constituent un défi tout particulier et sont de la plus grande importance (voir les directives de l'Académie Suisse des Sciences Médicales [4]).

\section{Prise en charge adéquate}

La personne âgée a droit jusqu'à la fin de sa vie à une prise en charge et à un traitement adaptés. L'âge, la dépendance, la perte d'autonomie ne doivent pas mener à une abstention de mesures thérapeutiques.

\section{Information appropriée}

La personne âgée a le droit d'être informée hon- situation, sur sa prise en charge médicale et sur les soins nécessaires afin de pouvoir consentir librement à ces mesures de manière éclairée.

\section{Processus décisionnels}

Chez la personne âgée, les processus décisionnels doivent prendre en compte l'autodétermination, la dignité et l'évaluation subjective de la qualité de vie. De ce fait, une procédure décisionnelle obligatoire et des structures sont nécessaires [5]. Toute mesure ne peut être entreprise qu'avec le consentement libre et éclairé de la personne âgée informée et capable de discernement. En cas d'incapacité de discernement, chaque décision doit être prise en fonction de la volonté présumée et dans l'intérêt objectif de la personne âgée.

\section{Mesure de privation de liberté}

Les mesures de privation de liberté en faveur des personnes âgées dangereuses pour elles-mêmes ou pour autrui doivent rester des mesures exceptionnelles qui ne peuvent être appliquées que dans des conditions strictement définies [4].

\section{Assistance au suicide}

L'assistance au suicide n'est pas du ressort de l'activité médicale gériatrique. Il existe à cet effet les directives de l'Académie Suisse des Sciences Médicales auxquelles se réfèrent les membres de la SPSG [6]. nêtement, de manière appropriée et adaptée à la

\subsection{Méthodes de travail, organisation et bases fondamentales de prise en charge}

\subsubsection{L'évaluation gériatrique comme méthode de travail}

L'évaluation gériatrique multidimensionnelle est un processus diagnostique interdisciplinaire complet (c'est-à-dire un processus d'investigagroupes professionnels différents) dont le but est d'identifier systématiquement les problèmes médicaux, fonctionnels et psychosociaux des personnes âgées ainsi que leur environnement physique afin de pouvoir les appréhender et établir un plan général de traitement et de prise en charge tenant compte des réalités personnelles de la vie et des besoins des patients. Ainsi, pour être efficace, l'évaluation gériatrique est toujours liée à une planification de la prise en charge (GEM: geriatric evaluation and management).c, d

L'évaluation gériatrique compréhensive est en effet la méthode de travail gériatrique la plus importante. L'examen du patient est approfondi et l'évaluation de ses problèmes de santé aux plans physique, psychique, social, fonctionnel, économique et spirituel est renforcée au même tion approfondi qui requiert la participation de 
titre que les relations à l'intérieur de l'équipe de soins responsable du diagnostic et du traitement.

En fonction des besoins, divers instruments d'évaluation standardisés et validés sont utilisés qui ne servent pas seulement au diagnostic et à la prise en charge mais qui permettent aussi de mesurer les conséquences des prises de décisions et de procéder à leur évaluation. Les conditions nécessaires pour un contrôle de la qualité et de la recherche sont ainsi mises en place.

\subsubsection{Le travail d'équipe interdisciplinaire}

La complexité du patient gériatrique et sa multidimensionnalité (santé physique, santé mentale, santé sociale, situation économique, indépendance, valeurs personnelles) rendent indispensable la constitution d'une équipe interdisciplinaire (gériatrie, soins, travail social, physio- et ergothérapie, aumôneries, psychologie, psychiatrie, etc.) afin d'établir un diagnostic et de fixer des objectifs ainsi que pour assurer la prise en charge et l'évaluation.

Chaque membre de cette équipe est compétent dans son domaine professionnel et possède des connaissances étendues sur l'activité des autres groupes professionnels. Un espace d'échange régulier d'informations structurées est mis sur pieds pour discuter, décider, coordonner et contrôler ensemble les objectifs de soins et la stratégie de prise en charge.

\subsubsection{Aspects organisationnels}

\subsubsection{Différences culturelles,} particularités régionales et cantonales

Le développement de l'offre de soins et de prise en charge des personnes âgées doit prendre en compte l'existence antérieure de structures de valeur et doit les inclure en respectant les diversités culturelles et régionales souvent issues du passé historique et profondément ancrées dans la population. D'ailleurs, plusieurs formes d'organisation sont imaginables pour une prise en charge optimale des personnes âgées.

\subsubsection{Intégration des structures de soins spécifiquement gériatriques}

Pour faire face aux besoins spécifiques et aux situations particulières des personnes âgées, des offres de prise en charge typiquement gériatrique sont nécessaires comme par exemple la prévention des chutes, le dépistage et le diagnostic de la démence ainsi que des programmes de renforcement de la santé et de prévention, des programmes ambulatoires de soins et des programmes de réadaptation.
Tout cela doit être intégré dans des structures générales qui travaillent avec d'autres fournisseurs de prestations et plus particulièrement avec les médecins de premier recours.

\subsubsection{Coordination et information}

Une coordination entre les différentes structures ambulatoires et hospitalières est nécessaire de même qu'un échange efficient d'information afin de garantir un maximum de continuité et de qualité dans la prise en charge.

L'offre de prise en charge entre les différentes structures hospitalières et ambulatoires doit être transparente et perméable. En effet, les personnes âgées se trouvent souvent dans un état de santé labile et elles ont à chaque fois besoin d'une structure de prise en charge adaptée à leur situation actuelle; des chaînes thérapeutiques sont donc nécessaires comme celle qui relie par exemple la gériatrie aiguë, la clinique de jour, la prise en charge par le médecin à domicile, le médecin de famille, l'établissement médico-social, la prise en charge par les soins à domicile, etc.

\subsubsection{Prestation d'ordre médical}

La prestation médicale dans une institution de gériatrie reconnue est effectuée par un médecin spécialiste titulaire de la formation approfondie FMH en gériatrie. La plupart des gériatres qui travaillent actuellement dans de telles institutions exercent toutefois également leurs activités dans un cabinet privé.

Les institutions reconnues pour la formation médicale postgraduée en gériatrie se répartissent en cliniques A et cliniques B. Ce sont des services bien définis sur le plan organisationnel dont l'objectif principal est la prise en charge gériatrique par des équipes interdisciplinaires. Les cliniques A sont dirigées par un gériatre à plein temps et les cliniques B par un médecin titulaire de la formation approfondie en gériatrie employé au minimum à mi-temps. Alors que les cliniques $\mathrm{A}$ proposent aussi bien une prise en charge gériatrique aiguë hospitalière qu'une prise en charge ambulatoire et de long séjour, les cliniques B proposent au moins deux de ces trois domaines d'activité. Des critères de reconnaissance plus élaborés figurent dans le programme de formation postgraduée de médecine interne qui inclut la formation approfondie de gériatrie et dans celui de médecine générale qui inclut aussi la formation approfondie de gériatrie (www.fmh.ch).

\subsubsection{Principes fondamentaux de la prise en charge des personnes âgées}

Les directives de l'Académie Suisse des Sciences Médicales sur la prise en charge des personnes 
âgées en situation de dépendance [4] s'adressent aux médecins, aux soignants et aux autres thérapeutes. Elles en mentionnent les principes fondamentaux: exigence d'une prise en charge appropriée, personnelle et continue des personnes âgées; travail en commun avec le domaine social et travail interdisciplinaire; valorisation de la formation prégraduée, postgraduée et continue des professionnels concernés.

\section{Structures gériatriques et processus de soins}

\subsection{Principes fondamentaux}

Le travail gériatrique consiste en un travail d'équipe structurée et interdisciplinaire. La conduite des équipes thérapeutiques est une des tâches primordiales du gériatre. Les membres de l'équipe proviennent de groupes professionnels tels que les soignants, les travailleurs sociaux, les thérapeutes (physio-, ergo- et activo-thérapeute, logopédiste), les psychologues, les aumôniers, les diététiciens ainsi que d'autres professionnels consultants sollicités au besoin.

\subsection{Gériatrie aiguë hospitalière (stationnaire)}

Les tâches de la gériatrie aiguë consistent en l'établissement du diagnostic et au traitement des personnes âgées malades qui nécessitent des soins hospitaliers en raison de comorbidités aiguës d'origine somatique ou psychique avec pour objectif premier la réintégration du patient dans son propre contexte social.

Les services ou les cliniques de gériatrie aiguë traitent de façon prédominante des personnes invalides très âgées qui présentent en général des maladies et des handicaps multidimensionnels. Ils intègrent systématiquement une prise en charge interdisciplinaire et des mesures précoces de réadaptation ce qui les distingue des institutions habituelles de médecine aiguë.

\subsection{Réadaptation gériatrique}

\subsubsection{Principes généraux}

La réadaptation dans le sens du maintien ou de l'amélioration de l'indépendance dans les activités de la vie quotidienne est le principe de base du traitement gériatrique et se trouve présente dans toutes les institutions gériatriques. Cette méthode est appliquée de façon très individualisée.

D'une part, il convient de prendre en compte les limites physiologiques et psycho-cognitives et d'autre part d'avoir recours aux ressources individuelles de la manière la plus optimale possi- ble. Ainsi, le concept de réadaptation devient dynamique, global et en même temps individuel et il est concentré sur les capacités restantes, les ressources et les souhaits individuels.

Eu égard à des patients le plus souvent très âgés et invalides, les mesures de réadaptation seront souvent interrompues par des problèmes et des complications médicales aigus, ce qui oppose le décours linéaire et limité dans le temps de la médecine aiguë à celui de la réadaptation.

La physiothérapie, l'ergothérapie, la logopédie, la thérapie active, la neuropsychologie, le conseil social et les soins de réadaptation travaillent ensemble dans un réseau serré. Les phases du processus de réadaptation comprennent aussi d'autres étapes: évaluation, planification, traitements, soins du patient et conseils à l'entourage, évaluation des résultats.

A côté des efforts de réadaptation pour récupérer, maintenir ou améliorer la maîtrise des activités quotidiennes figurent des programmes de réadaptation spécifique pour certaines affections comme par exemple après un accident vasculaire cérébral ou une fracture de la hanche.

Les principes énoncés ci-dessus sont bien sûr valables pour tous les groupes d'âge; toutefois, en réadaptation gériatrique, une durée adéquate doit être concédée au patient déficitaire en raison de la diminution de ses réserves fonctionnelles et de la réduction de sa capacité de résistance.

\subsubsection{Réadaptation gériatrique hospitalière (stationnaire)}

La réadaptation gériatrique se préoccupe du traitement du handicap et des capacités fonctionnelles sur les bases éprouvées de la classification de l'OMS pour la récupération, la stabilisation, et l'acquisition d'un décours de vie au grand âge le plus indépendant possible dans un environnement donné. Les groupes concernés sont ceux qui ont perdu tout ou partie de leur indépendance ou de leur habileté à la suite le plus souvent de nombreuses maladies ou d'accidents ce qui a entraîné des répercussions sur leur vie sociale (participation) compte tenu de leur contexte de vie passée et de leur mode de vie (facteurs contextuels personnels et environnementaux).

\subsection{Prise en charge de transition}

La prise en charge de transition a pour but la sortie rapide de l'hôpital aigu ou constitue une alternative limitée de courte durée à l'hospitalisation dans des situations bien définies où la problématique médicale est connue et la stabilité médicale du patient assurée dans lesquelles le soin gériatrique, le traitement psychosocial et 
l'avis médical doivent être garantis comme par exemple dans le cadre d'un service de consultations.

L'offre de prestation comprend justement l'avis, le traitement, le soin et la prise en charge afin d'éviter une hospitalisation en urgence ou de permettre de supporter le poids des situations psychosociales antérieures ou nouvelles rencontrées lors de décompensations répétées du réseau social. La stabilisation du patient et de son environnement en constitue donc le projet.

En fonction du potentiel disponible, la prise en charge de transition doit permettre d'atteindre à nouveau le degré d'indépendance antérieure ou de maintenir ce degré après un certain laps de temps grâce à des soins et des traitements actifs dans le sens de la prévention et de mesures visant à prévenir la survenue de nouvelles dépendances encore plus importantes.

\subsection{Prise en charge de longue durée en établissement de long séjour ou en EMS}

Il s'agit des personnes qui nécessitent des soins impossibles à donner de manière ambulatoire et dont le maintien ou le retour à domicile n'est pas dans leur meilleur intérêt. Leur prise en charge doit donc pouvoir être poursuivie dans des institutions de soins de longue durée. Dans certaines situations, il peut être sensé d'entrer plus tôt dans une institution de longue durée pour renforcer par exemple l'intégration psychosociale.

Avant l'entrée en institution de longue durée, une évaluation gériatrique interdisciplinaire doit être assurée pour que, malgré une prise en charge ambulatoire adéquate ou après des investigations en gériatrie aiguë, un traitement et une réadaptation hospitalière, cette décision corresponde bien à la meilleure option médico-sociale pour la personne âgée et pour sa famille compte tenu des ressources financières individuelles. Pour cette raison, l'Académie Suisse des Sciences Médicales recommande aussi une évaluation gériatrique multidimensionnelle [4].

L'objectif de la gériatrie en soins de longue durée est de maintenir et de renforcer les compétences et les capacités physiques, psychiques et sociales des personnes en fonction de la représentation individuelle de la qualité de vie. Les institutions de longue durée ont un devoir professionnel de garantir un traitement et une prise en charge gériatriques dont la qualité soit contrôlée. formation approfondie pour les médecins spécialistes en psychiatrie et psychothérapie qui se spécialisent en psychiatrie et psychothérapie de la personne âgée. Elle contient une formation postgraduée d'une durée de 2 ans en psychiatrie et psychothérapie de la personne âgée.

\subsection{Prise en charge palliative \\ Sous les termes de prise en charge palliative (pal-} liative care en anglais), on entend un traitement et une prise en charge globale des personnes souffrant de maladies inguérissables, de maladies aiguës ou de maladies chroniques avancées menaçant leur vie (voir [12]).

Le but d'une prise en charge palliative est de permettre que les patients et leur entourage jouissent d'une qualité de vie aussi bonne que possible. Pour cette raison, les symptômes pénibles doivent être soulagés de manière optimale et, en fonction des souhaits du patient, les aspects sociaux, spirituels et religieux seront également prise en compte.

Le point fort des soins palliatifs réside dans le temps, celui dans lequel le fait de mourir et la mort elle-même seront prévisibles. C'est pourquoi il est fondamental de mettre en place ces soins suffisamment tôt, en parallèle avec des mesures curatives ou de réhabilitation, car, en gériatrie, il n'existe pas toujours de séparation nette entre les essais curatifs et palliatifs.

Les soins palliatifs en gériatrie sont pratiqués par une équipe multidisciplinaire et nécessitent des connaissances approfondies en médecine palliative pour ne pas agir de façon nihiliste ou au contraire trop agressive.

Les discussions difficiles portant sur les traitements en fin de la vie, sur le renoncement au traitement ou l'arrêt des traitements, sur la prise de décision chez le patient incapable de discernement appartiennent donc bien aux tâches de la gériatrie.

\subsection{Prise en charge géronto-psychiatrique}

Les gériatres ont l'obligation d'accomplir une formation postgraduée additionnelle en gérontopsychiatrie au cours de laquelle ils apprennent à s'occuper des personnes souffrant de démence et de dépression ainsi que d'affections psychiques comme la confusion, la peur, les hallucinations et la dépendance (addiction). Dans les situations difficiles, ils se référeront au consultant gérontopsychiatre et pourront leur transmettre la prise en charge de certains patients. ${ }^{\mathrm{e}}$

Le but de la prise en charge géronto-psychiatrique consiste en la restauration ou l'amélioration de la santé mentale dans le cadre le plus souvent d'une multimorbidité et d'une situation sociale qui conditionne aussi le contexte général. Ainsi, l'information, le conseil et le soutien social sont des éléments importants pour trouver une solution appropriée. Le ressenti par rapport aux forces perdues, aux limites fonctionnelles et aux causes des pertes principales sont précisément des problèmes psychiques difficiles chez le patient en gériatrie.
3.8 Prévention et renforcement de la santé Les prestations de renforcement de la santé et de prévention des maladies comprennent toutes les 
mesures qui ont pour but de maintenir ou d'améliorer l'état de santé des patients. Elles préviennent aussi bien la survenue de nouvelles maladies qu'elles en assurent le traitement sur le plan des capacités fonctionnelles et de la participation à la société. Ces mesures comprennent surtout les préventions primaires et secondaires.

Les mesures de prévention seront établies en pratique comme suit: les mesures prophylactiques dont le succès n'est pas immédiatement attendu concernent les personnes âgées avec une espérance de vie conséquente. D'autres mesures préventives, dont le succès attendu doit être immédiat comme la vaccination contre la grippe sont destinées à la plus grande partie des personnes âgées [13].

Une tâche d'importance croissante, basée sur des preuves, consiste en la réduction préventive des besoins en soins pendant la vieillesse, en collaboration étroite avec le médecin de famille ou avec le médecin de premier recours et les organisations de soins à domicile au travers de la visite préventive à domicile [14].

\subsection{Gériatrie ambulatoire}

Les gériatres sont aujourd'hui compétents dans les domaines ambulatoires suivants:

\subsubsection{Gériatrie en médecine} de premier recours et en soins à domicile Les gériatres sont capables d'une part d'être des médecins de premier recours et ils sont d'autre part aussi capables d'assumer des tâches de conseils et d'enseignement.

\subsubsection{Clinique mémoire}

Cela comprend les investigations interdisciplinaires, le traitement et le conseil aux patients qui souffrent d'affections cérébrales, respectivement de symptômes liés aux démences. L'activité de conseil concerne évidemment aussi l'entourage. Ainsi, une attention particulière sera portée sur les affections et sur les perturbations psychiques ainsi que sur les aides complémentaires telles que les offres de décharge comme les séjours temporaires, les groupes d'aide pour l'entourage, les services d'aides et de soins à domicile, etc.

\subsubsection{Clinique des chutes}

La chute et les causes de vertige seront analysées et identifiées au moyen de l'anamnèse, de tests cliniques et d'explorations fonctionnelles. Un programme individuel d'entraînement sera mis en place pour exercer la force et l'équilibre. Au besoin, la situation individuelle à domicile sera étudiée de manière à prévenir les risques de chute.

\subsubsection{Clinique de l'incontinence}

Les investigations et le traitement de l'incontinence urinaire seront effectués, cas échéant avec l'aide des appareils diagnostiques gynécologiques et urologiques. Le conseil thérapeutique, l'utilisation des aides et au besoin le réentraînement du plancher pelvien seront proposés.

\subsubsection{Clinique de jour}

La prise en charge partiellement hospitalière dans le contexte d'une clinique de jour est partie intégrante et nécessaire des offres de prestation gériatrique de réadaptation afin de poursuivre des traitements hospitaliers, de raccourcir la durée du traitement hospitalier, d'éviter ou de retarder une hospitalisation. La clinique de jour est un maillon du réseau de traitement gériatrique.

Le raccourcissement de la durée physiologique nécessaire à la convalescence du patient gériatrique constitue la base du besoin de temps additionnel nécessaire à la récupération des capacités fonctionnelles pour le maintien de l'indépendance pendant la vieillesse.

\subsubsection{Séjour de répit, hôpital de jour, séjour intermédiaire}

Les séjours de répit sont des séjours limités dans le temps effectués dans une institution de soins pour des patients âgés vivant à domicile nécessitant une prise en charge; ils servent à décharger les personnes soignantes de l'entourage.

Les séjours d'un jour ou d'une nuit constituent des offres de prise en charge ambulatoire pour une seule journée ou une seule nuit de la semaine destinée à des personnes âgées vivant à la maison; ils servent en premier lieu à décharger les personnes soignantes de l'entourage.

Les séjours intermédiaires sont des séjours limités dans le temps en institution de soins pour personnes âgées dépendantes qui ne peuvent être prodigués à domicile jusqu'à ce qu'elles puissent être admises dans l'institution de leur choix.

Pour toutes ces offres de soins, les compétences et les ressources physiques, psychiques et sociales des personnes concernées doivent être maintenues et renforcées sous la direction d'un(e) gériatre ou avec le conseil d'un(e) gériatre.

\section{Références}

1 Schoenenberger A, Stuck AE. Health care for older persons in Switzerland: a country profile. J Am Geriatr Soc. 2006;54:986-90.

2 Weiterbildungsprogramm zum Facharzt für Innere Medizin bzw. Allgemeinmedizin, speziell Geriatrie. www.fmh.ch. 
3 Fisher AL. Just what defines frailty? J Americ Geriatric Soc. 2005;53:2229-30.

4 Académie Suisse des Sciences Médicales. Traitement et prise en charge des personnes âgées en situation de dépendance (2004). www.samw.ch.

5 Académie Suisse des Sciences Médicales. Droit des patientes et patients à l'autodétermination (2005). www.samw.ch.

6 Académie Suisse des Sciences Médicales. Prise en charge des patientes et patients en fin de vie (2004). www.samw.ch.

7 Rubenstein LZ, Stuck AE. Multidimensional Geriatric Assessment. In: Pathy MSJ, Sinclair AJ, Morley JE (eds.). Principles and Practice of Geriatric Medicine. $4^{\text {th }}$ edition. Hoboken NJ: John Wiley \& Sons, Ltd. 2006; p. 1543-52.

8 Ellis G, Langhorne P. Comprehensive geriatric assessment for older hospital patients. Br Med Bull. 2005; $71: 43-57$

9 Stuck AE, Siu AL, Wieland GD, et al. Comprehensive geriatric assessment: meta-analysis of controlled trials. Lancet. 1993;342:1032-6.
10 Cohen HJ, Feussner JR, Weinberger M, et al. A controlled trial of inpatient and outpatient geriatric evaluation and management. N Engl J Med. 2002;365(12):905-12.

11 Campion ED. Specialised care for elderly patients: improvement in quality of life without increase in costs. N Engl J Med. 2002;346(12):874.

12 Académie Suisse des Sciences Médicales. Soins palliatifs. Directives et recommandations médicoéthiques. ASSM 2006. www.samw.ch.

13 Seematter B, et al. Revue Méd Suisse. 2003; 123(11):665-9.

14 Stuck AE, Egger M, Hammer A, Minder CE, Beck JC. Home visits to prevent nursing home admission and functional decline in elderly people: Systematic review and meta-regression analysis. JAMA. 2002;287:1022-8. 